\title{
Dielectric Relaxation and Hopping Conduction in $\mathrm{La}_{2} \mathrm{NiO}_{4+\delta}$
}

\author{
Woo-Hwan Jung \\ Division of Electrical Engineering, Howon University, 64, Howondae 3 gil, Impi, Gunsan 573-930, Republic of Korea \\ Correspondence should be addressed to Woo-Hwan Jung; jungwoohwan@gmail.com
}

Received 27 November 2012; Accepted 18 January 2013

Academic Editor: Iwan Kityk

Copyright (C) 2013 Woo-Hwan Jung. This is an open access article distributed under the Creative Commons Attribution License, which permits unrestricted use, distribution, and reproduction in any medium, provided the original work is properly cited.

\begin{abstract}
An ac conductivity as well as dielectric relaxation property of $\mathrm{La}_{2} \mathrm{NiO}_{4.1}$ is reported in the temperature range of $77 \mathrm{~K}-130 \mathrm{~K}$ and in the frequency range of $20 \mathrm{~Hz}-1 \mathrm{MHz}$. Complex impedance plane plots show that the relaxation (conduction) mechanism in this material is purely a bulk effect arising from the semiconductive grain. The relaxation mechanism has been discussed in the frame of electric modulus spectra. The scaling behavior of the modulus suggests that the relaxation mechanism describes the same mechanism at various temperatures. The logarithmic angular frequency dependence of the loss peak is found to obey the Arrhenius law with the activation energy of $\sim 0.09 \mathrm{eV}$. The frequency-dependent electrical data are also analyzed in the frame of ac conductivity formalism. The ac conductivity has been found to follow a power-law behavior at a limited temperature and frequency region where Anderson localization plays a significant role in the transport mechanism for $\mathrm{La}_{2} \mathrm{NiO}_{4.1}$.
\end{abstract}

\section{Introduction}

Perovskite oxides with high dielectric constant play an important role in microelectronics [1] and have been used as memory devices and capacitors. In the literature, the dielectric relaxation with extremely high permittivity has been reported in many materials, in which the contribution of conduction carriers to dielectric polarization may play an important role. However, the explanation of this phenomenon is controversial based on different models $[2,3]$. The electron localized on an ion can cause displacements of neighboring ions from their positions in the crystal lattice. The quasi-particle formed by an electron and corresponding lattice displacements is called polaron $[4,5]$.

Crystal chemical considerations indicate that among the ternary transition metal oxides of the type $\mathrm{A}_{2} \mathrm{BO}_{4}$, only $\mathrm{La}_{2} \mathrm{NiO}_{4}$ should be stable in the $\mathrm{K}_{2} \mathrm{NiF}_{4}$ structure [6-8]. The natures and properties of $\mathrm{La}_{2} \mathrm{NiO}_{4}$ are highly sensitive to the content of excess oxygen. In fact, $\mathrm{La}_{2} \mathrm{NiO}_{4+\delta}$ exhibits the metal-insulator transition [8]. Although $\mathrm{La}_{2} \mathrm{NiO}_{4+\delta}$ remains semiconducting nature up to $\delta=0.25$, hole doping due to excess oxygen induces increasing structural distortion and changes electronic structures which modify the band gap width [9]. $\mathrm{La}_{2} \mathrm{NiO}_{4+\delta}$ exhibits semiconducting behavior at low temperatures with thermally activated or variable range hopping-type conductivity $[10,11]$. The previous experimental results suggest a high possibility that the major carrier at low temperature in $\mathrm{La}_{2} \mathrm{NiO}_{4+\delta}$ is a polaron $[10,11]$. However, the details of the transport kinetics are still unknown. Although there are many ways of addressing polaron dynamics, dielectric relaxation measurements are useful metrics because the hopping process of polaron has a high probability of involving a dielectric relaxation process [12-16]. Furthermore, many perovskite materials have shown high dielectric or ferroelectric properties and the dipole unit in these systems plays an important role in the electronic transport phenomena $[17,18]$.

In this paper, we investigate the electrical relaxation properties of $\mathrm{K}_{2} \mathrm{NiF}_{4}$ type $\mathrm{La}_{2} \mathrm{NiO}_{4+\delta}$ ceramic in the temperature range $77 \mathrm{~K}-300 \mathrm{~K}$ and frequency rang $20 \mathrm{~Hz}-1 \mathrm{MHz}$ by means of dielectric spectroscopy. These studies may give valuable information on the electrical properties, which are very important for practical applications of perovskite oxides.

\section{Experimental}

$\mathrm{La}_{2} \mathrm{NiO}_{4+\delta}$ polycrystalline ceramic was prepared by conventional solid state synthesis technique. $\mathrm{La}_{2} \mathrm{O}_{3}(>99.99 \%$ purity) and $\mathrm{NiO}(>99.9 \%$ purity) powders were used. First, the mixed powder was heated in air at $1273 \mathrm{~K}$ for $12 \mathrm{~h}$. After being ground and mixed well, the powder was heated again in air at $1373 \mathrm{~K}$ for $12 \mathrm{~h}$. Then the powder was pressed into the pellets and 


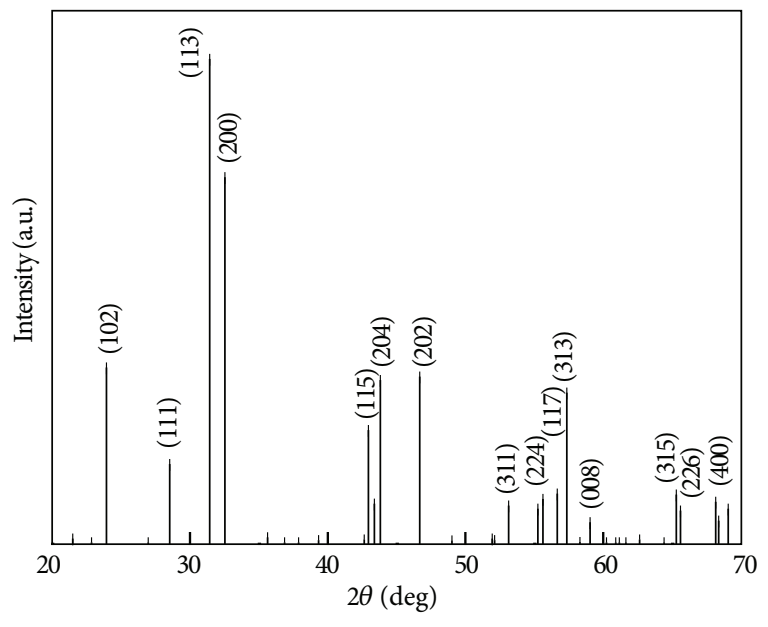

Figure 1: X-ray diffraction pattern of $\mathrm{La}_{2} \mathrm{NiO}_{4.1}$.

sintered finally in pure flowing oxygen at $1423 \mathrm{~K}$ for $12 \mathrm{~h}$. The powder diffraction using a $\mathrm{Cu} \mathrm{K} \alpha \mathrm{X}$-ray indicates the tetragonal structure with the lattice constants $a=3.8615$, $c=12.6484 \AA$. Figure 1 shows the XRD spectrum of the sintered $\mathrm{La}_{2} \mathrm{NiO}_{4}$ sample recorded at room temperature. The oxygen content of the sample was determined by iodometric titration. The experimental error of the oxygen content is within $\pm 3 \%$. $\delta$ is found to be 0.1 . The chemical composition was confirmed by inductively coupled plasma (ICP). The composition was found to be nearly identical to the nominal one within the accuracy of ICP.

Capacitance and impedance were obtained as functions of temperature by four-terminal pair ac impedance measurement method using a HP 4284A precision LCR meter. The measured values of capacitance and impedance were correlated by calibrating capacitance and resistance of leads to zero. Flat surfaces of the specimens were coated with an In-Ga alloy in the ration $7: 3$ by a rubbing technique for an electrode.

\section{Results and Discussion}

Figure 2 shows the frequency dependence of real part $\left(Z^{\prime}\right)$ of impedance of $\mathrm{La}_{2} \mathrm{NiO}_{4.1}$ as a function of temperature. In Figure 2, $Z^{\prime}$ decreases with an increase in frequency for all temperatures implies relaxation process in the specimens. The magnitude of $Z^{\prime}$ decreases at lower frequencies with an increasing temperature which indicates increase in the ac conductivity and this can be also attributed to the fact that $Z^{\prime}$ values decrease with increase of temperature indicating reduction of grains, grain boundaries, and electrode interface resistance. The merge of $Z^{\prime}$ values at higher frequencies is observed, which may be due to the release of space charge $[19,20]$.

Figures 3(a) and 3(b) show the frequency dependence of imaginary $\left(Z^{\prime \prime}\right)$ part of impedance of $\mathrm{La}_{2} \mathrm{NiO}_{4.1}$ as a function of temperature. The pattern of variation is characterized by the occurrence of peaks with asymmetric broadening which indicates relaxation in the materials. Double peaks are observed at $130 \mathrm{~K}$. The peaks in higher and lower frequency

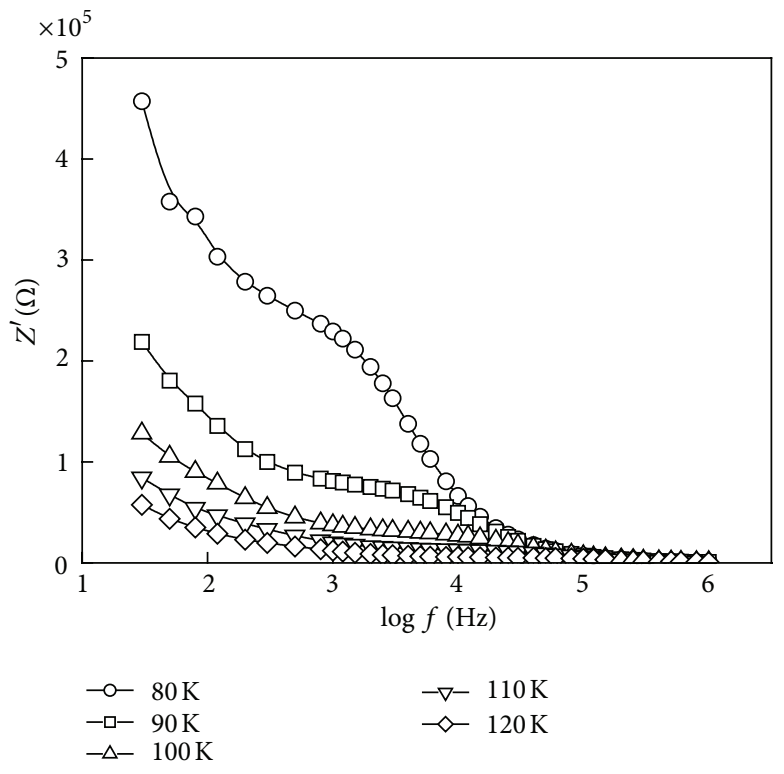

FIGURE 2: Frequency dependence of the real part $\left(Z^{\prime}\right)$ of impedance at various temperatures for $\mathrm{La}_{2} \mathrm{NiO}_{4.1}$.

regions are found due to effect of bulk and grain boundaries, respectively. Thus, the observed lower frequency region peaks above $140 \mathrm{~K}$ are due to the effect of grain boundaries. The peak values of $Z^{\prime \prime}$ are observed to decrease with increasing temperature. It indicates an increase of loss in the resistive property of the materials. Shifting of peaks toward the higher frequency sides with increasing temperatures indicates the temperature dependence of the relaxation time. Furthermore, the broadening of the peaks with rise in temperature indicates nonunique relaxation timescale, whose variance/distribution widens at higher frequency side $[19,21]$.

Figure 4 represents the variation of $\mathrm{Z}^{\prime \prime} / \mathrm{Z}_{\max }^{\prime \prime}$ with $\log \left(f / f_{\max }\right)$ at different temperatures. It shows the scaling behavior of the materials. The overlap of the curves for all the temperatures into a single master curve indicates that relaxation described the same mechanism at different temperatures in this material.

We have adopted the modulus formalism to study the relaxation mechanism. The usefulness of the modulus representation in the analysis of the relaxation properties has been demonstrated for poly crystalline ceramics. The advantage of adopting complex electric modulus formalism is that it can discriminate against electrode polarization and grain boundary conduction mechanism [22]. It is also suitable in detecting bulk phenomena properties as apparent conductivity relaxation time [23]. The other advantage of the electric modulus is that the electrode effect can be suppressed [23]. Electric modulus corresponds to the relaxation of electric field in the material when the electric displacement remains constant. Thus the electric modulus represents the real dielectric relaxation process. The complex electric modulus $M^{*}=M^{\prime}+j M^{\prime \prime}$ can be expressed as [24]

$$
M^{*}(\omega)=M_{\infty}\left[1-\int_{0}^{\infty}\left(\frac{-d \phi(t)}{d t}\right) \exp (-j \omega t) d t\right]
$$




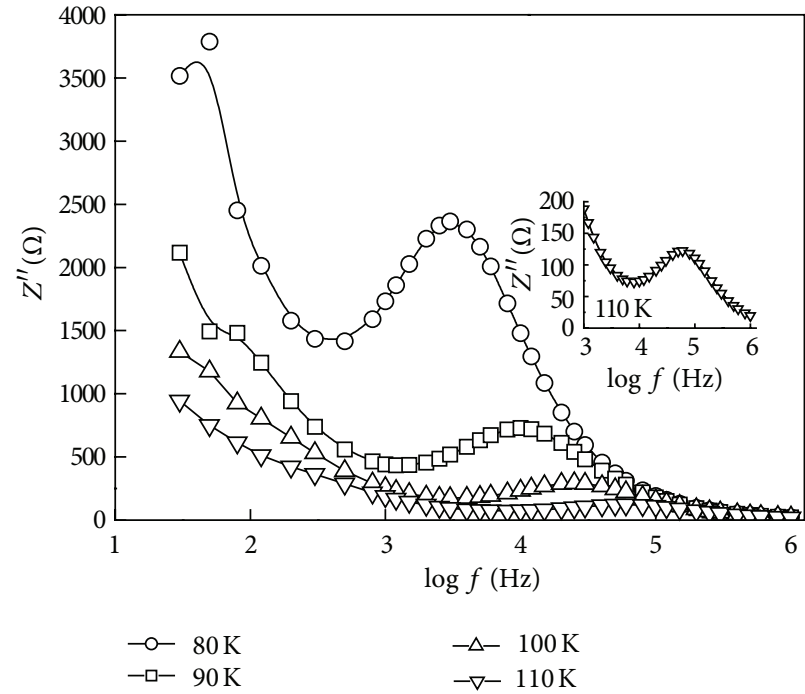

(a)

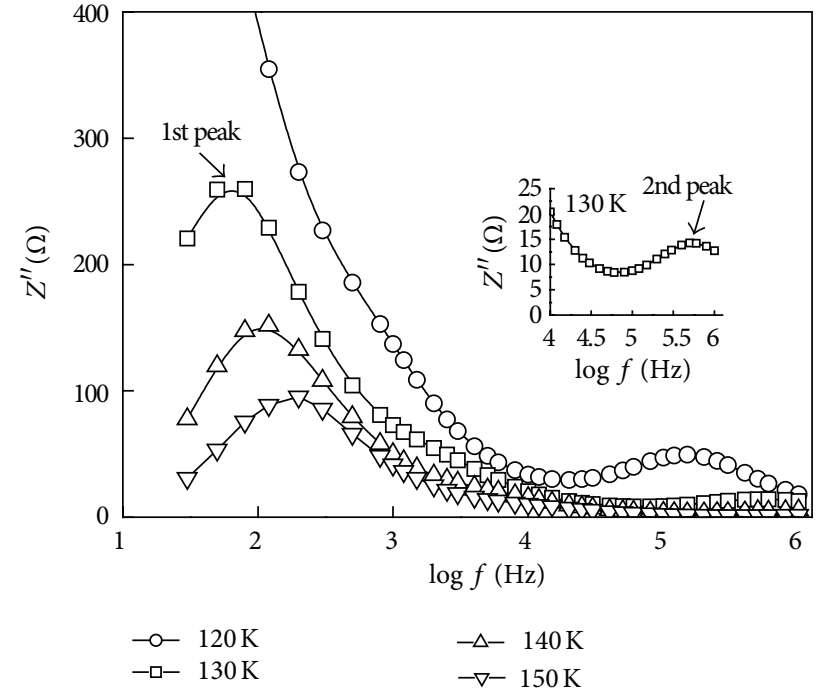

(b)

FIGURE 3: Frequency dependence of the imaginary part $\left(Z^{\prime \prime}\right)$ of impedance at various temperatures for $\mathrm{La}_{2} \mathrm{NiO}_{4.1}$; (a) $T<120 \mathrm{~K}$ (b) $T>120 \mathrm{~K}$.

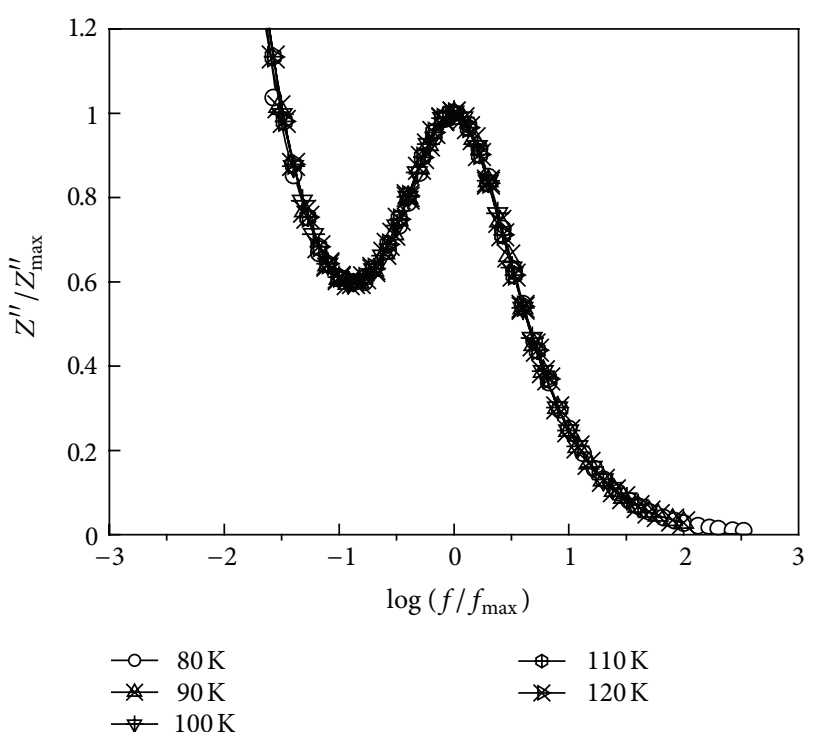

FIGURE 4: Scaling behavior of $Z^{\prime \prime}$ at various temperatures for $\mathrm{La}_{2} \mathrm{NiO}_{4.1}$.

where $M^{\prime}$ and $M^{\prime \prime}$ are real and imaginary part of $M^{*} . \omega=$ $2 \pi f$ is the angular frequency, $M_{\infty}=1 / \varepsilon_{\infty}$ is the asymptotic value of $M^{\prime}(\omega)$, and $\phi(t)=\exp \left[-\left(t / \tau_{M}\right) \beta\right]$ represents time evolution of the electric field within the material where $\beta(0<\beta<1)$ is the stretched exponent and $\tau_{M}$ is the conductivity relaxation times. The $M^{*}$ formalism is widely used to study conductivities of materials. The variation of imaginary component $M^{\prime \prime}$ as a function of frequency at different temperatures provides useful information of the charge transport mechanism such as electrical transport and conductivity relaxation process. Peaks are observed in the frequency spectra of $M^{\prime \prime}$ which indicate the existence of conductivity relaxation process. The frequencies at which the peaks in $M^{\prime \prime}$ spectroscopic plots are observed follow the relation $\omega_{\max } \tau_{m}=1$, where $\omega_{\max }$ is the angular frequency corresponding to the peak maximum. It is typically observed that $\tau_{m}$ follows the Arrhenius law given by $[22,23]$

$$
\tau_{M}=\tau_{M 0} \exp \left(E / k_{B} T\right),
$$

where $\tau_{M 0}$ is the preexponential factor and $E$ is the activation energy.

The frequency dependence of $M^{\prime}(\omega)$ is shown in Figure 5(a). At low frequency, the value of $M^{\prime}(\omega)$ decreases as the temperature increases. Also at a given temperature, $M^{\prime}(\omega)$ increases with increase in the frequency and takes almost a constant value at higher frequency. At low frequency and in the high temperature region, $M^{\prime}(\omega)$ approaches zero, confirming an appreciable electrode and/or ionic polarization [25]. Figure 5(b) shows the variation in $M^{\prime \prime}(\omega)$ with frequency at different temperatures where the peaks are shifted toward higher frequencies with increasing temperature. We have fitted the relaxation times $\left(\tau_{m}\right)$ obtained from the peak frequencies with inverse of temperature according to the Arrhenius law given by (2) where the fit is shown by solid line in inset of Figure 6. It is observed that the value of relaxation time is found to be decreasing with increasing temperature which indicates semiconductor behavior of the compound. For a typical lattice dipole relaxation, $\tau_{M 0}$ is on the order of $10^{-10} \sim 10^{-13} \mathrm{~s}$ [18]. The value of $E$ and $\tau_{M 0}$ obtained from the Arrhenius fit is $E \approx 0.09 \mathrm{eV}$ and $\tau_{M 0} \approx 2 \times 10^{-10} \mathrm{~s}$. This experimental result indicates that the dielectric loss anomaly (polaron-like relaxation) observed in this compound is arising from the interaction between free charges and lattice, which induces the local dipole. The lattices distortions introduced by the excess oxygen and associated vacancies are sufficient to produce more than one off-center equilibrium position for $\mathrm{Ni}^{3+}$ ions. The observed relaxation is associated with thermal activation 


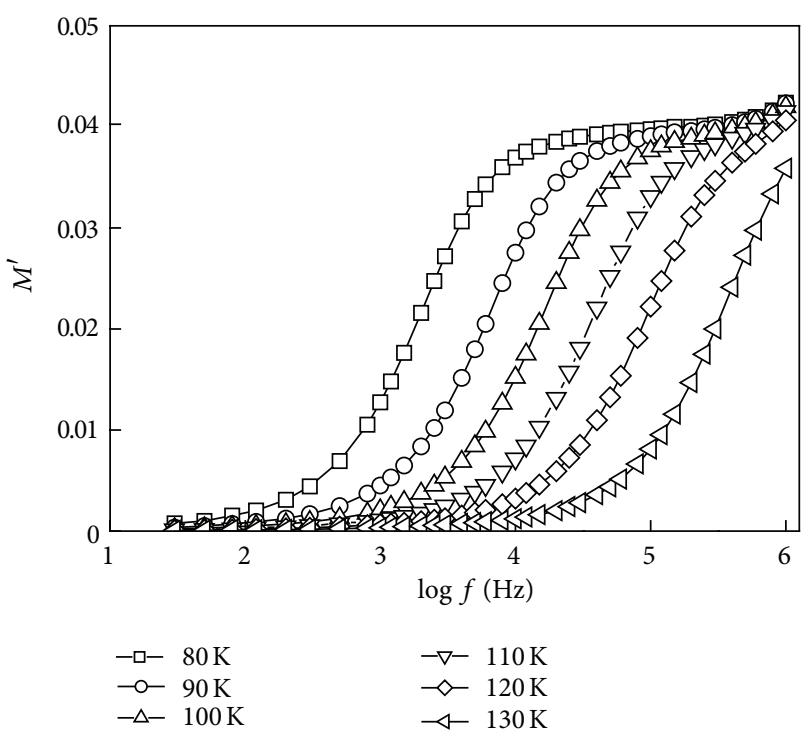

(a)

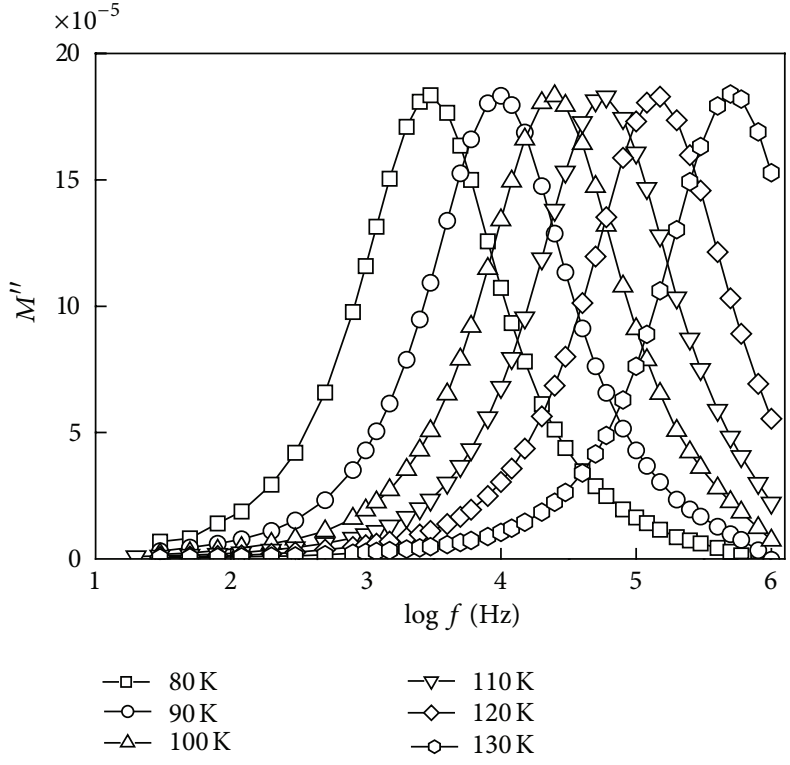

(b)

Figure 5: (a) Frequency dependence of the real part $\left(M^{\prime}\right)$ of electric modulus at various temperatures for $\mathrm{La}_{2} \mathrm{NiO}_{4.1}$. (b) Frequency dependence of imaginary part $\left(M^{\prime \prime}\right)$ of electric modulus at various temperatures for $\mathrm{La}_{2} \mathrm{NiO}_{4.1}$.

motion between these equivalent potential minima [26]. Under the action of an ac field, the charge may hop between equivalent available lattice sites depending on the crystal symmetry. This hopping is equivalent to the reorientation of an electric dipole, which gives frequency-dependent, complex, dielectric properties exhibiting Debye-type behavior. Thus the experimental results suggest that spectral intensity of the electric modulus observed in $\mathrm{La}_{2} \mathrm{NiO}_{4.1}$ is thermally activated like dielectric relaxation occurring in the other various materials in which the process of hopping of small polaron dominates the electrical transport [18, 20, 26-28].

Scaling of the electric modulus can give further information about the dependence of the relaxation dynamics on the temperature, structure, and also on the concentration of the charge carrier [29]. Figure 6 shows the scaling results at different temperatures where $M_{\max }^{\prime \prime}$ and $\left(f / f_{\max }\right)$ are used as the scaling parameters for $M^{\prime \prime}$ and $f$, respectively. Clearly all modulus spectra can be seen to completely overlap and are scaled to a single master curve indicating that relaxation dynamics does not change with temperature.

In Figure 7, the variation of normalized parameter $\left(M^{\prime \prime} / M_{\max }^{\prime \prime}\right)$ and $\left(\tan \delta / \tan \delta_{\max }\right)$ as a function of logarithmic angular frequency measured at $100 \mathrm{~K}$ is shown. The overlapping peak position of $\left(M^{\prime \prime} / M_{\max }^{\prime \prime}\right)$ and $\left(\tan \delta / \tan \delta_{\max }\right)$ curves is an evidence of delocalized or long-range relaxation. However, for the present system the $\left(M^{\prime \prime} / M_{\max }^{\prime \prime}\right)$ and $\left(\tan \delta / \tan \delta_{\max }\right)$ peaks do not overlap but are very close suggesting the components from both long-range and localized relaxation [29].

Figure 8 shows the variation of ac conductivity $\left(\sigma_{\mathrm{ac}}\right)$ as a function of frequency at different temperatures. It is obvious that the conductivity increases with increasing frequency and increasing temperature. The observed behavior is typical of the existence of polarons in the system $[13,16-19,30]$.
The very basic fact about ac conductivity in complex perovskite oxides is that conductivity is an increasing function of frequency (any hopping model has this feature). Andersonlocalized charge carriers contribute to conductivity by hopping processes which lead to an increase in complex conductivity with frequency $(\omega)$. This behavior can be described by using a power law with exponent $s<1$ which is usually represented as "universal dielectric response" (UDR) [31-33]. By considering a dc conductivity term, the real part of the ac conductivity is described as

$$
\sigma(\omega)=\sigma(0)+A \omega^{s},
$$

where $\sigma(\omega)$ is the total conductivity, $\sigma(0)$ the frequency independent conductivity, that is, dc conductivity, $A$ depends on the temperature, and $s$ is the dimensionless frequency exponent. In case of measurement at sufficiently high frequency, a crossover from power law, $\sigma \propto \omega^{s}$, to a linear increase of $\omega, \sigma \propto \omega$, is frequently observed in the variety of materials which is defined as the "second universality" (SU) [31-33]. The microscopic picture of the origin of SU region at high frequency is still not established.

It is seen from Figure 8 that at low frequency a step-like feature is observed possibly due to the grain boundary effect where a corresponding relaxation peak is observed in the $Z^{\prime \prime}$ spectrum. With further increasing frequency, a crossover to the linear $\sigma(\omega) \propto \omega$ dependence above $\sim 10^{5} \mathrm{~Hz}$ is observed. Linearity of the frequency dependence is shown by broken line in the figure. The linear SU region is shifted toward higher frequencies with increasing temperature and consequently the SU region is shifted out of the frequency window above $130 \mathrm{~K}$. The UDR and SU contributions to the intrinsic ac conductivity in the present investigation are consistent with results for single crystal or polycrystalline of $\mathrm{LaMnO}_{3}[13,18]$. 


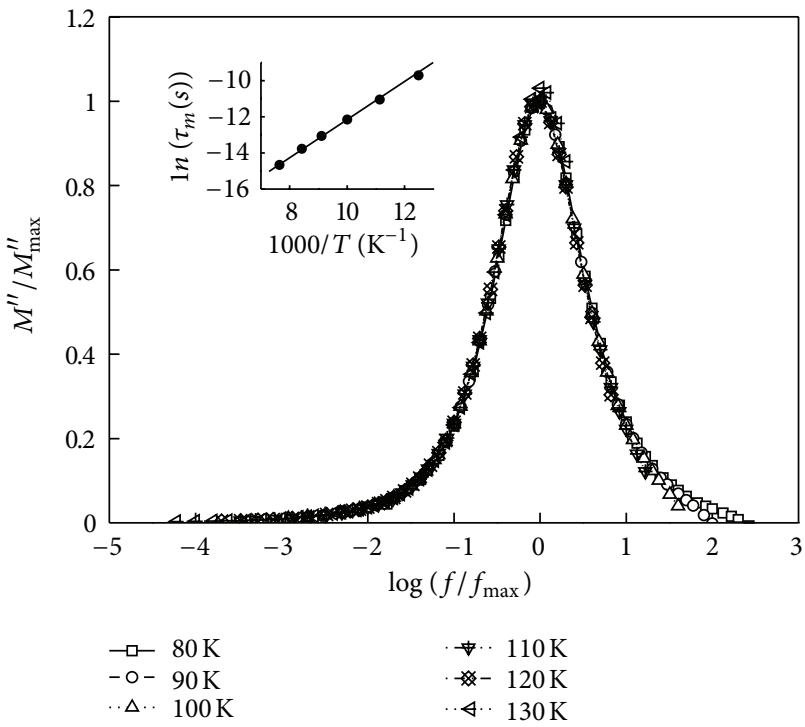

FIGURE 6: Scaling behavior of $M^{\prime \prime}$ at various temperatures for $\mathrm{La}_{2} \mathrm{NiO}_{4.1}$. The temperature dependence of the most probable relaxation time $\left(\tau_{m}\right)$ obtained from the frequency-dependent plot of $M^{\prime \prime}$ is shown in the inset where the symbols are the experimental data and the solid line is the least-squares straight line fit.

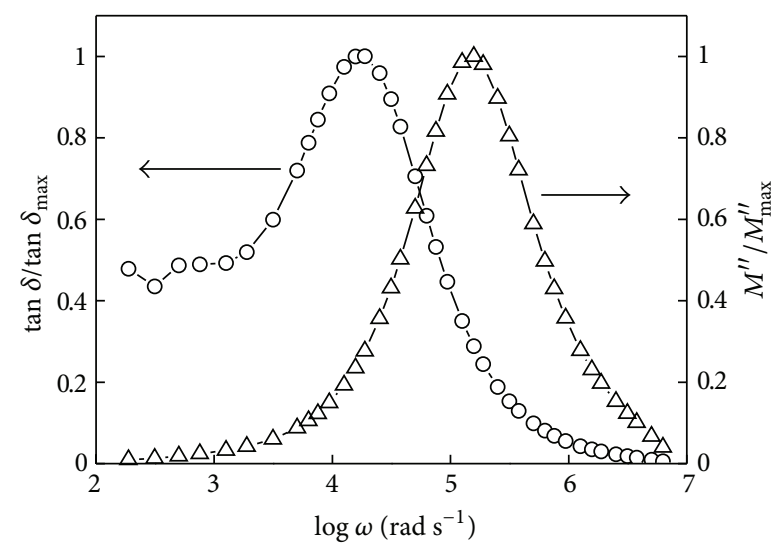

FIgURE 7: Logarithmic angular frequency dependence of normalized peaks. $\tan \delta / \tan \delta_{\max }$ and $M^{\prime \prime} / M_{\max }^{\prime \prime}$ for $\mathrm{La}_{2} \mathrm{NiO}_{4.1}$ at $100 \mathrm{~K}$.

For $\mathrm{La}_{2} \mathrm{NiO}_{4.1}, \sigma(\omega)$ increases with increasing frequency and therefore the observed ac conductivity must be related to the bound carriers trapped in the specimen. The frequency dependence of ac conductivity at various temperatures resembles that of hopping-type conduction between these trap centers. $\mathrm{Ni}^{3+}$ is one kind of charge trap. Hopping of charge carriers among the trap leaves situated in the band gap of the material may give rise to a frequency dependence ac conductivity with a frequency dependence $\sim \omega^{s}(s<1)$. Since UDR contribution to the ac conductivity is found in a very small region of the frequency, a large error is noted while analyzing the ac conductivity by power law for $\mathrm{La}_{2} \mathrm{NiO}_{4.1}$. The temperature dependence of the frequency exponent $s$ is shown in the inset of Figure 8. For $\mathrm{La}_{2} \mathrm{NiO}_{4.1}, s$ varies between 1.0 and 0.8 . There are vast numbers of theoretical

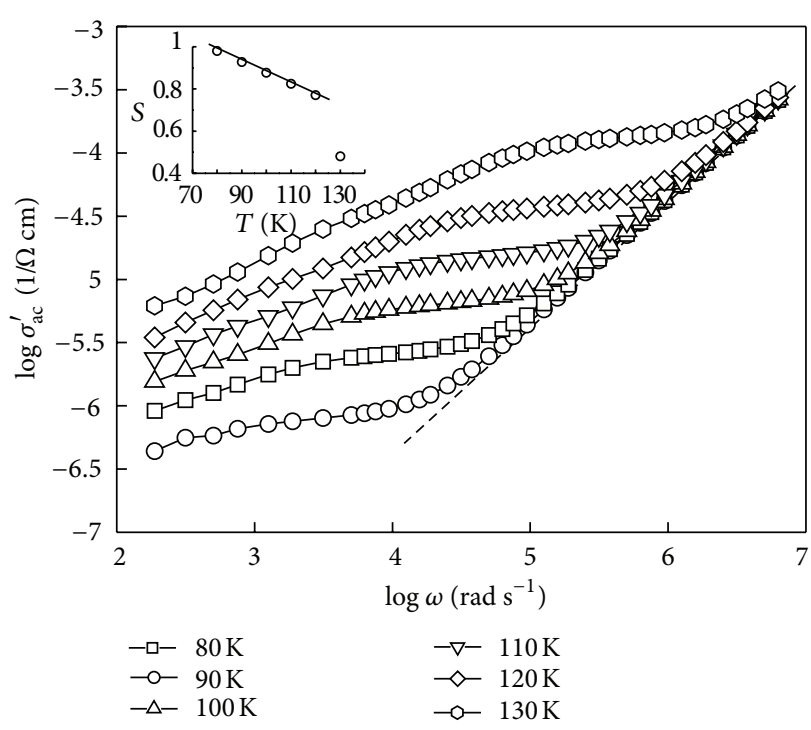

FIGURE 8: Frequency dependence of ac conductivity at some selected temperature. Inset: the temperature dependence of the exponent parameter, $s$.

approaches to deduce this behavior form the microscopic transport properties of various classes of materials [34]. The small polaron tunneling (SPT) model, which corresponds to the variable range hopping model for nonzero frequencies, predicts a temperature-independent frequency exponent $s$ near 0.8 [31-33]. The results of a previous experiment showed that the $\mathrm{dc}$ conduction mechanism of $\mathrm{La}_{2} \mathrm{NiO}_{4+\delta}$ is well described by variable range hopping conduction $[10,11]$. This suggests that the SPT model is an appropriate mechanism for the low temperature ac conductivity of the present materials. Unfortunately, due to the small frequency range where the $\omega^{s}$ power law is seen, an unambiguous determination of ac conduction mechanism is difficult but the overall response is very similar to that found for Sr-doped $\mathrm{LaMnO}_{3}[13,18]$ which is a well-described SPH model.

\section{Conclusions}

The frequency dependence of electric modulus, impedance, and ac conductivity of $\mathrm{La}_{2} \mathrm{NiO}_{4.1}$ ceramic synthesized by solid-state reaction method is investigated in the temperature range from $80 \mathrm{~K}$ to $130 \mathrm{~K}$ and frequency range $20 \mathrm{~Hz}$ to $1 \mathrm{MHz}$. The relaxation mechanism has been discussed in the framework of electric modulus. The shifting of the peak position to the higher frequency side with increasing temperature indicates the polaron transport in this material. The logarithmic angular frequency dependence of loss peak is found to obey the Arrhenius law with the activation energy of $0.09 \mathrm{eV}$. This value of activation energy suggests that the bulk conduction maybe due to polaron hopping based on electron carriers. The scaling behavior of the electric modulus and impedance suggest that relaxation mechanism describes the same mechanism at various temperatures. The frequencydependent ac conductivity data are also analyzed in the framework of conductivity formalism. 


\section{Acknowledgment}

This work was supported by Howon University.

\section{References}

[1] R. J. Cava, "Dielectric materials for applications in microwaves communications," Journal of Materials Chemistry, vol. 11, no. 1, pp. 54-62, 2001.

[2] S. Komine, "Dielectric relaxation study on $\mathrm{Ce}_{0.9} \mathrm{Gd}_{0.1} \mathrm{O}_{1.95}$ ceramics," Physica B, vol. 392, no. 1-2, pp. 348-352, 2007.

[3] E. Iguchi and K. J. Lee, "Dielectric relaxations in $\mathrm{SrTiO}_{3}$ doped with $\mathrm{La}_{2} \mathrm{O}_{3}$ and $\mathrm{MnO}_{2}$ at low temperatures," Journal of Materials Science, vol. 28, no. 21, pp. 5809-5813, 1993.

[4] E. Iguchi, H. Satoh, H. Nakatsugawa, and F. Munakata, "Correlation between hopping conduction and transferred exchange interaction in $\mathrm{La}_{2} \mathrm{NiO}_{4+\delta}$ below $300 \mathrm{~K}$," Physica B, vol. 270, no. 3-4, pp. 332-340, 1999.

[5] C. N. R. Rao, D. J. Buttrey, N. Otsuka et al., "Crystal structure and semiconductor-metal transition of the quasi-twodimensional transition metal oxide, $\mathrm{La}_{2} \mathrm{NiO}_{4}$, Journal of Solid State Chemistry, vol. 51, no. 2, pp. 266-269, 1984.

[6] K. K. Singh, P. Ganguly, and J. B. Goodenough, "Unusual effects of anisotropic bonding in $\mathrm{Cu}(\mathrm{II})$ and $\mathrm{Ni}(\mathrm{II})$ oxides with $\mathrm{K}_{2} \mathrm{NiF}_{4}$ structure," Journal of Solid State Chemistry, vol. 52, no. 3, pp. 254-273, 1984.

[7] M. Sayer and P. Odier, "Electrical properties and stoichiometry in $\mathrm{La}_{2} \mathrm{NiO}_{4}$," Journal of Solid State Chemistry, vol. 67, no. 1, pp. 26-36, 1987.

[8] D. C. Johnston, J. P. Stokes, D. P. Goshorn, and J. T. Lewandowski, "Influence of oxygen defects on the physical properties of $\mathrm{La}_{2} \mathrm{CuO}_{4-y}$," Physical Review B, vol. 36, no. 7, pp. 4007-4010, 1987.

[9] Th. Strangfeld, K. Westerholt, and H. Bach, "Electronic charge transport and magnetism of the system $\left(\mathrm{La}_{1-x} \mathrm{Sr}_{x}\right)_{2} \mathrm{NiO}_{4+\delta}$," Physica C, vol. 183, no. 1-3, pp. 1-10, 1991.

[10] T. Katsufuji, T. Tanabe, T. Ishikawa, Y. Fukuda, T. Arima, and Y. Tokura, "Optical spectroscopy of the charge-ordering transition in $\mathrm{La}_{1.6} \mathrm{Sr}_{0.33} \mathrm{NiO}_{4}$, Physical Review B, vol. 54, no. 20 , pp. R14230-R14233, 1996.

[11] J. M. Bassat, J. P. Loup, and P. Odier, "Progressive change with $\mathrm{T}$ from hopping to random phase propagation in $\mathrm{La}_{2-x} \mathrm{NiO}_{4-\delta}$," Journal of Physics Condensed Matter, vol. 6, no. 40, pp. 82858293, 1994.

[12] A. Mansingh, J. M. Reyes, and M. Sayer, "Dielectric behaviour in a vanadium phosphate glass," Journal of Non-Crystalline Solids, vol. 7, no. 1, pp. 12-22, 1972.

[13] A. Seeger, P. Lunkenheimer, J. Hemberger et al., "Charge carrier localization in $\mathrm{La}_{1-x} \mathrm{Sr}_{x} \mathrm{MnO}_{3}$ investigated by ac conductivity measurements," Journal of Physics Condensed Matter, vol. 11, no. 16, pp. 3273-3290, 1999.

[14] H. Jhans, D. Kim, R. J. Rasmussen, and J. M. Honig, "acconductivity measurements on $\mathrm{La}_{2} \mathrm{NiO}_{4+\delta}$," Physical Review $B$, vol. 54, no. 16, pp. 11224-11229, 1996.

[15] W. H. Jung, J. H. Sohn, J. H. Lee, J. H. Sohn, M. S. Park, and S. H. Cho, "Alternating-current electrical properties of $\mathrm{CaMnO}_{3}$ below the Neel temperature," Journal of the American Ceramic Society, vol. 83, no. 4, pp. 797-801, 2000.

[16] C. Ang, Z. Yu, Z. Jing, P. Lunkenheimer, and A. Loidl, "Dielectric spectra and electrical conduction in Fe-doped $\mathrm{SrTiO}_{3}$," Physical Review B, vol. 61, no. 6, pp. 3922-3926, 2000.
[17] G. Chern, W. K. Hsieh, M. F. Tai, and K. S. Hsung, "High dielectric permittivity and hole-doping effect in $\mathrm{La}_{1-x} \mathrm{Sr}_{x} \mathrm{FeO}_{3}$," Physical Review B, vol. 58, no. 3, pp. 1252-1260, 1998.

[18] A. Karmakar, S. Majumdar, and S. Giri, "Polaron relaxation and hopping conductivity in $\mathrm{LaMn}_{1-x} \mathrm{Fe}_{x} \mathrm{O}_{3}$," Physical Review $B$, vol. 79, no. 9, pp. 094406-094413, 2009.

[19] M. Ram, "A.c. Conductivity and dielectric properties of $\mathrm{LiNi}_{3 / 5} \mathrm{Cu}_{2 / 5} \mathrm{VO}_{4}$ ceramics," Physica B, vol. 405, no. 5, pp. 13591361, 2010.

[20] Y. Q. Lin, Y. J. Wu, X. M. Chen, S. P. Gu, J. Tong, and S. Guan, "Dielectric relaxation mechanisms of $\mathrm{BiMn}_{2} \mathrm{O}_{5}$ ceramics," Journal of Applied Physics, vol. 105, no. 5, Article ID 054109, 2009.

[21] R. S. Kumar and K. Hariharan, "AC conductivity and electrical relaxation studies on $10 \mathrm{CuI}-60 \mathrm{AgI}-30 \mathrm{~V}_{2} \mathrm{O}_{5}$ glasses," Materials Chemistry and Physics, vol. 60, no. 1, pp. 28-38, 1999.

[22] P. K. Bajpai and K. N. Singh, "Dielectric relaxation and ac conductivity study of $\mathrm{Ba}\left(\mathrm{Sr}_{1 / 3} \mathrm{Nb}_{2 / 3}\right) \mathrm{O}_{3}$," Physica $B$, vol. 406, no. 6-7, pp. 1226-1232, 2011.

[23] P. Kumar, B. P. Singh, T. P. Sinha, and N. K. Singh, "AC conductivity and dielectric relaxation in $\mathrm{Ba}\left(\mathrm{Sm}_{1 / 2} \mathrm{Nb}_{1 / 2}\right) \mathrm{O}_{3}$ ceramic," Physica B, vol. 406, no. 2, pp. 139-143, 2011.

[24] H. Mahamoud, B. Louati, F. Hlel, and K. Guidara, "Impedance and modulus analysis of the $\left(\mathrm{Na}_{0.6} \mathrm{Ag}_{0.4}\right)_{2} \mathrm{PbP}_{2} \mathrm{O}_{7}$ compound," Journal of Alloys and Compounds, vol. 509, no. 20, pp. 60836089, 2011.

[25] D. K. Mahato, A. Dutta, and T. P. Sinha, "Impedance spectroscopy analysis of double perovskite $\mathrm{Ho}_{2} \mathrm{NiTiO}_{6}$," Journal of Materials Science, vol. 45, no. 24, pp. 6757-6762, 2010.

[26] W. H. Jung, "Investigations of the charge transport properties in (LaMn) ${ }_{0.956} \mathrm{O}_{3}$ at low temperatures," Journal of Physics Condensed Matter, vol. 18, no. 29, pp. 6691-6698, 2006.

[27] M. Idrees, M. Nadeem, and M. M. Hassan, "Investigation of conduction and relaxation phenomena in $\mathrm{LaFe}_{0.9} \mathrm{Ni}_{0.1} \mathrm{O}_{3}$ by impedance spectroscopy," Journal of Physics D, vol. 43, no. 15, Article ID 155401, 2010.

[28] A. Dutta and T. P. Sinha, "Impedance spectroscopy study of $\mathrm{BaMb}_{1 / 3} \mathrm{Nb}_{2 / 3} \mathrm{O}_{3}$ : frequency and time domain analyses," Physica B, vol. 405, no. 6, pp. 1475-1479, 2010.

[29] S. Saha and T. P. Sinha, "Low-temperature scaling behavior of $\mathrm{BaFe}_{0.5} \mathrm{Nb}_{0.5} \mathrm{O}_{3}$," Physical Review B, vol. 65, no. 13, Article ID 134103, pp. 1341031-1341037, 2002.

[30] A. Levstik, C. Filipič, V. Bobnar, S. Drnovšek, J. Holc, and M. Kosec, "Polaron conductivity mechanism in $0.5 \mathrm{~Pb}\left(\mathrm{Zr}_{0.5} 75 \mathrm{Ti}_{0.425}\right) \mathrm{O}_{30.5} \mathrm{~Pb}\left(\mathrm{Fe}_{2 / 3} \mathrm{~W}_{1 / 3}\right) \mathrm{O}_{3}$," Physica B, vol. 405 , no. 20, pp. 4271-4273, 2010.

[31] S. R. Elliott, "A.c. conduction in amorphous chalcogenide and pnictide semiconductors," Advances in Physics, vol. 36, no. 2, pp. 135-218, 1987.

[32] W. K. Lee, J. F. Liu, and A. S. Nowick, "Limiting behavior of ac conductivity in ionically conducting crystals and glasses: a new universality," Physical Review Letters, vol. 67, no. 12, pp. 15591561, 1991.

[33] A. S. Nowick, A. V. Vaysleyb, and B. S. Lim, "Further evidence for a "second universality" in alternating-current conductivity relaxation," Journal of Applied Physics, vol. 76, no. 7, pp. 44294431, 1994.

[34] A. R. Long, "Frequency-dependent loss in amorphous semiconductor," Advances in Physics, vol. V 31, no. 5, pp. 553-637, 1982. 

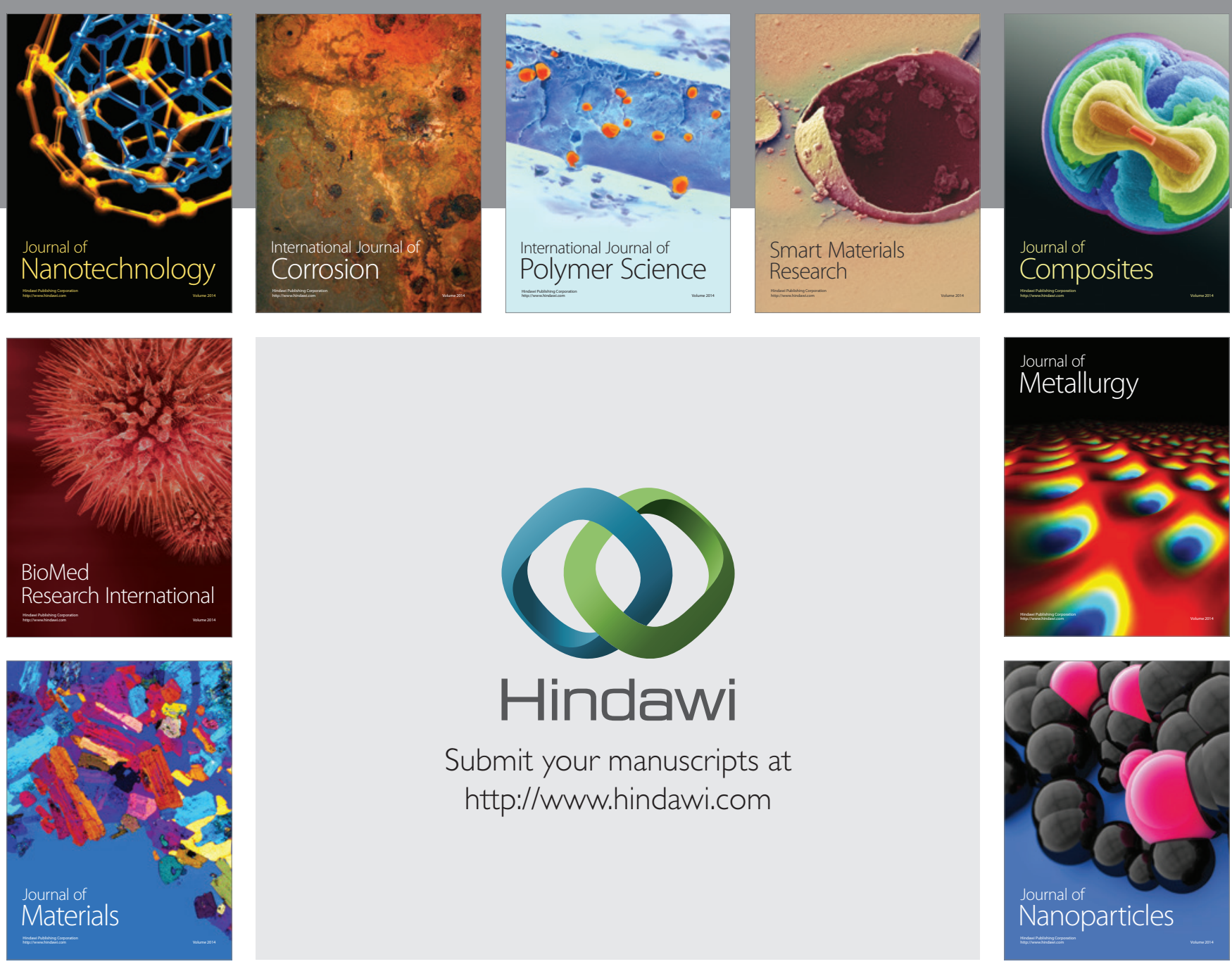

Submit your manuscripts at http://www.hindawi.com
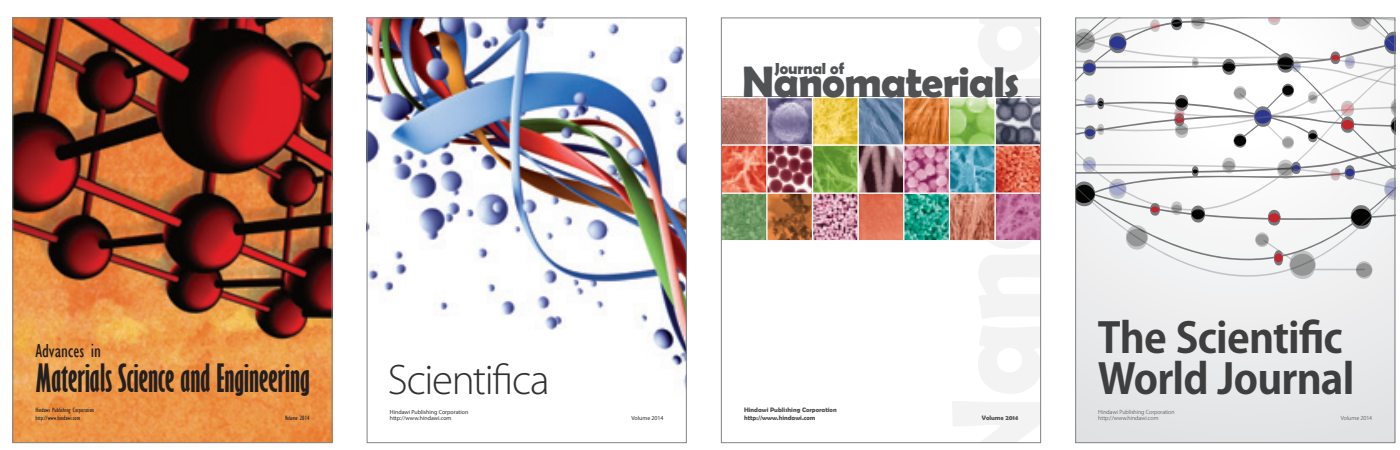

\section{The Scientific World Journal}
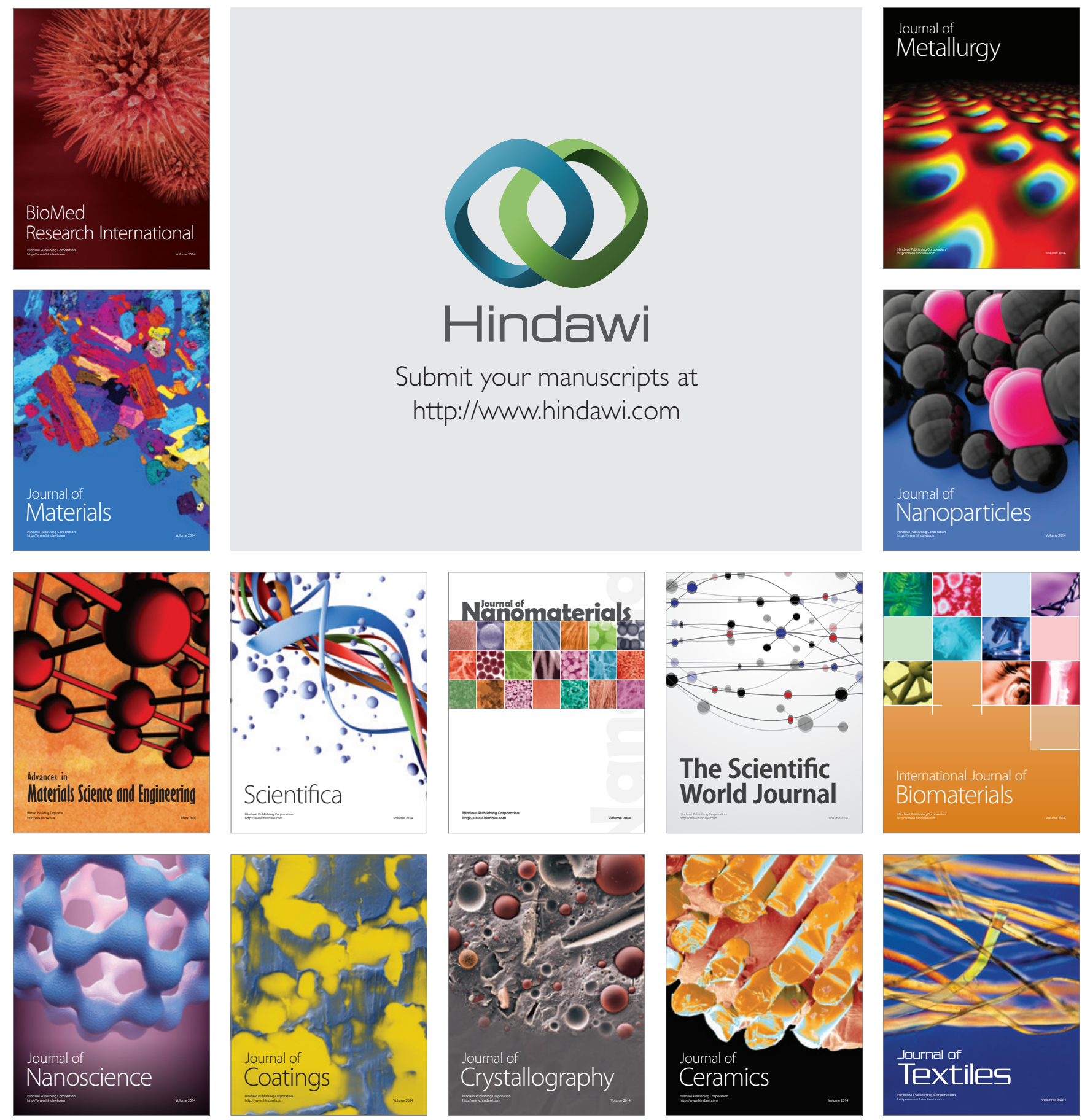